\title{
Discrete Morse inequalities on infinite graphs
}

\author{
Rafael Ayala, Luis M. Fernández and José A. Vilches * \\ Departamento de Geometría y Topología \\ Facultad de Matemáticas, Universidad de Sevilla, Sevilla, SPAIN \\ rdayala@us.es, lmfer@us.es, vilches@us.es
}

Submitted: Jul 19, 2007; Accepted: Mar 9, 2009; Published: Mar 20, 2009

Mathematics Subject Classification: 05C10, 57M15

\begin{abstract}
The goal of this paper is to extend to infinite graphs the known Morse inequalities for discrete Morse functions proved by R. Forman in the finite case. In order to get this result we shall use a special kind of infinite subgraphs on which a discrete Morse function is monotonous, namely, decreasing rays. In addition, we shall use this result to characterize infinite graphs by the number of critical elements of discrete Morse functions defined on them.
\end{abstract}

\section{Introduction.}

Under a classical point of view, Morse theory looks for links between global properties of a smooth manifold and critical points of a function defined on it (see [5]). For instance, the so-called Morse inequalities relate the Betti numbers of the manifold and the numbers of such critical points. R. Forman [1] introduced the notion of discrete Morse function defined on a finite $c w$-complex and, in this combinatorial context, he developed a discrete Morse theory as a tool for studying the homotopy type and homology groups of these complexes. He also proved the corresponding Morse inequalities, analogous to the classical ones obtained in the smooth case. This theory has shown to have many applications (for instance, see [2]).

Following Forman's suggestions stated in [1], we have begun the study of discrete Morse functions on infinite simplicial complexes by establishing the extension of Morse inequalities which allow us to understand the relationships between the topology of the complex, critical simplices of a discrete Morse function defined on it and the monotonous behaviour of this function at the ends of the complex. The authors made this work for

${ }^{*}$ The authors are partially supported by Plan Nacional de Investigación 2.007, Project MTM200765726, SPAIN, 2007 and P.A.I. project 2008/FQM-189, SPAIN, 2008. 
the special case of non-compact connected surfaces without boundary in [3]. In this paper, we deal with one-dimensional complexes in a more general way, by obtaining Morse inequalities for any kind of graph. Under this point of view, new situations arise, when finite Morse inequalities do not hold, which are fully understood by taking into account the criticality of the considered function at the infinite.

In Section 1, we recall basic Forman's definitions of discrete Morse function and critical simplex as well as his discrete Morse inequalities for the finite case. Besides, we state some general results concerning graphs which we shall use later. In Section 2, we study discrete Morse functions on infinite graphs. First, we give some examples which point out that new situations appear with respect to the finite case, specially discrete Morse functions without critical simplices and we prove that trees are the only infinite graphs in which it is possible to define such special discrete Morse functions. In order to investigate these new situations, we introduce the notion of weakly decreasing rays which becomes essential in the rest of the paper. Section 3 includes our main result: the discrete Morse inequalities which relate the topology of an infinite graph and the number of critical simplices of a discrete Morse function defined on it by using the number of weakly decreasing rays, generalizing Forman's result for the finite case. Finally, in Section 4 we give an application of the discrete Morse inequalities which consists on characterizing infinite connected graphs which admit a discrete Morse function with a fixed number of critical elements (critical simplices and decreasing rays).

\section{Preliminaries.}

Let $M$ be a simplicial complex. A discrete Morse function defined on $M$ is a function $f: M \longrightarrow \mathbb{R}$ such that, for any $p$-simplex $\sigma \in M$ :

(M1) At most one $(p+1)$-simplex $\tau$ containing $\sigma$ verifies $f(\tau) \leq f(\sigma)$.

(M2) At most one $(p-1)$-simplex $v$ contained in $\sigma$ verifies $f(v) \geq f(\sigma)$.

A $p$-simplex $\sigma \in M$ is said to be critical with respect to $f$ if:

(C1) There is not any $(p+1)$-simplex $\tau$ containing $\sigma$ satisfying $f(\tau) \leq f(\sigma)$.

(C2) There is not any $(p-1)$-simplex $v$ contained in $\sigma$ satisfying $f(v) \geq f(\sigma)$.

The above notions were introduced by R. Forman in [1] for the finite case and we shall use them for the infinite case with no changes. Then, we have, by a direct computation, the following proposition which holds for both finite and infinite cases:

Proposition 1.1. Let $f$ be a discrete Morse function defined on a simplicial complex $M$. Given a subcomplex $N \subseteq M$, the restriction of $f$ to $N$ is a discrete Morse function too. Moreover, if $\sigma \in N$ is a critical p-simplex with respect to $f$, then $\sigma$ is also a critical p-simplex with respect to the restriction of $f$ to $N$. 
Let us remind the reader that given a $c w$-complex $M$, the $p$-th Betti number of $M$, denoted by $b_{p}(M)$, is the rank of the $p$-th homology group of $M, H_{p}(M)$, with integer coefficients. In particular, $b_{0}(M)$ counts the number of connected components of $M$.

In [1], Forman also established the discrete Morse inequalities for discrete Morse functions defined on a finite simplicial complex:

Theorem 1.2. (Discrete Morse inequalities for finite complexes) Let $f$ be a discrete Morse function defined on a finite cw-complex $M$. Then:

(I1) $m_{p}(f)-m_{p-1}(f)+\cdots \pm m_{0}(f) \geq b_{p}(M)-b_{p-1}(M)+\cdots \pm b_{0}(M)$;

(I2) $m_{p}(f) \geq b_{p}(M)$

(I3) $m_{0}(f)-m_{1}(f)+m_{2}(f)-\cdots \pm m_{\operatorname{dim}(M)}(f)=b_{0}(M)-b_{1}(M)+b_{2}-\cdots \pm b_{\operatorname{dim}(M)}(M)$, where $m_{p}(f)$ denotes the number of critical p-simplices with respect to $f$.

Consequently, from (I2) we get that, for $p=0$, any discrete Morse function on any finite simplicial complex always has critical 0 -simplices.

Now, we are going to state, for later use, some general results concerning discrete Morse functions on either finite or infinite graphs, whose proofs are obtained directly from the definitions. Moreover, from now on, we shall assume the classic terminology of Graph Theory, that is, we shall write vertex instead of 0 -simplex and edge instead of 1-simplex. For more details and terminology concerning Graph Theory, see book of Diestel [4].

Proposition 1.3. Let $M$ be a graph, $N \subseteq M$ be a subgraph of $M$ and $f$ be a discrete Morse function defined on $M$. Then, any critical edge of $f$ in $N$ is a critical edge of $f$ in $M$.

Notice that the above proposition does not establish anything about critical vertices. In fact, it is easy to show that the number of critical vertices with respect to the restriction is, in general, greater than the number of critical vertices on the whole graph. For instance, we have the following example:

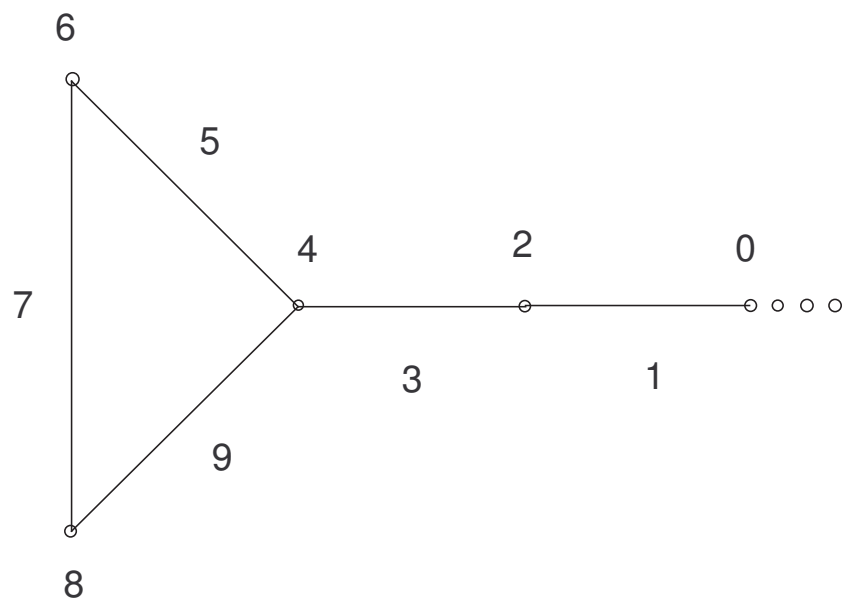


If we consider $N$ as the subgraph formed by the cycle, the vertex $f^{-1}(4)$ is a critical vertex of $N$, but it is not a critical vertex of $M$.

Definition 1.4. Let $M$ be a graph and $f$ be a discrete Morse function defined on $M$. Given a vertex $v \in M$, we say that $f(v)$ is a local minimum (resp. maximum) of $f$ in $M$ if $f(v)$ is less (resp. greater) than $f(e)$ where $e$ is any edge containing $v$. Similarly, given an edge $e \in M$, we say that $f(e)$ is a local maximum of $f$ in $M$ if $(e)$ is greater than $f(v)$ and $f(w)$ where $v$ and $w$ are the two vertices contained in $e$.

Notice that, by means of condition (M1), a vertex of degree at least 2 cannot be a local maximum. Moreover, condition (M2) implies that the value of $f$ on any edge $e$ must be greater than at least one of the values on its two vertices and thus $f(e)$ cannot be a local minimum.

In the next result the notion of critical simplex of a discrete Morse function on a graph is reformulated in terms of local extrema (maxima or minima) of the function.

Proposition 1.5. Let $M$ be a graph and $f$ be a discrete Morse function defined on $M$. Then, a vertex $v$ of $M$ is critical with respect to $f$ if and only if $f(v)$ is a local minimum of $f$ on $M$. Moreover, an edge $e$ of $M$ is a critical edge with respect to $f$ if and only if $f(e)$ is a local maximum of $f$ on $M$.

\section{Discrete Morse functions on infinite graphs.}

First, we present some examples which show that discrete Morse inequalities for the finite case (Theorem 1.2) do not hold in this new context:

Example 2.1. Non existence of critical simplices. Let $M$ be the graph of the following figure in which it is defined a discrete Morse function as described:

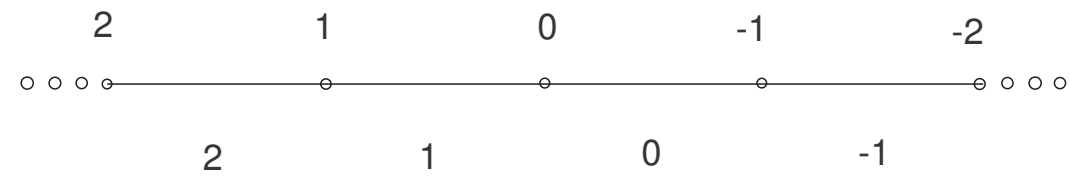

In this example, $m_{0}=0, m_{1}=0, b_{0}=1$ and $b_{1}=0$. Thus, (I2) and (I3) of Theorem 1.2 do not hold.

Example 2.2. Infinite number of critical simplices. Let $M$ be the graph of the following figure in which it is defined a discrete Morse function as described:

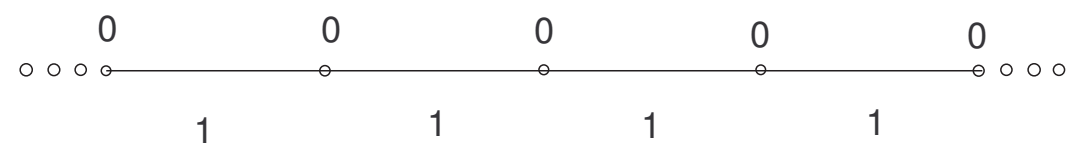


In this example $m_{0}=\infty, m_{1}=\infty, b_{0}=1$ and $b_{1}=0$. So, (I1) with $p=1$ and (I3) of Theorem 1.2 do not hold.

Example 2.3. Finite number of critical points. Let $M$ be the graph of the following figure in which it is defined a discrete Morse function as described:

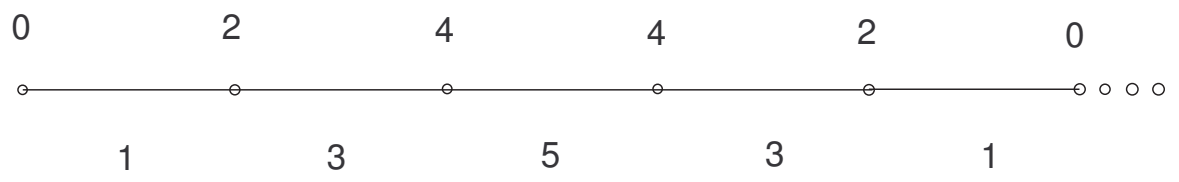

Here, $m_{0}=1, m_{1}=1, b_{0}=1$ and $b_{1}=0$. So, (I3) of Theorem 1.2 does not hold.

To study these new situations, a certain class of infinite subgraphs, the rays, will play an outstanding role. A ray of an infinite graph $M$ is an infinite connected subgraph $R \subseteq M$ such that all of vertices of $R$ have degree two but one $v_{0}$ which has degree one. Then, we say that $R$ is a ray starting at $v_{0}$. Usually we shall denote a ray $R$ by listing its vertices and edges in an ordered way:

$$
R=v_{0}, e_{0}, v_{1}, e_{1}, \ldots
$$

Given two rays in the same infinite graph, we say they are equivalent or cofinal if, from a common vertex, they coincide.

We say that a discrete Morse function $f$ is monotonous on a ray $R=v_{0}, e_{0}, v_{1}, e_{1}, \ldots$ if one of the following chain of inequalities it is verified:

(1) $f\left(v_{0}\right)<f\left(e_{0}\right) \leq f\left(v_{1}\right)<f\left(e_{1}\right) \leq f\left(v_{2}\right)<f\left(e_{2}\right) \leq \ldots$. In this case we say that $f$ is weakly increasing on $R$.

(2) $f\left(v_{0}\right) \geq f\left(e_{0}\right)>f\left(v_{1}\right) \geq f\left(e_{1}\right)>f\left(v_{2}\right) \geq f\left(e_{2}\right)>\ldots$. In this case we say that $f$ is weakly decreasing on $R$.

If all inequalities in (1) or (2) are strict we say that $f$ is either decreasing or increasing, respectively.

As we shall see later, weakly decreasing rays will play a central role in the generalized discrete Morse inequalities. We characterize them in the following proposition:

Proposition 2.4. Let $M$ be an infinite graph and let $f$ be a discrete Morse function defined on $M$. Given a ray $R$ in $M$, the following conditions are equivalent:

(i) $f$ is weakly decreasing on $R$.

(ii) There is not any critical simplex with respect to the restriction of $f$ to $R$, denoted by $f_{\mid R}$.

(iii) There is not any local minimum or maximum of $f_{\mid R}$ excluding, possibly, the first vertex of $R$, which could be the unique local maximum of $f_{\mid R}$. 
Proof. Let $R$ be the ray $v_{0}, e_{0}, v_{1}, e_{1}, \ldots, v_{n}, e_{n}, v_{n+1}, e_{n+1}, \ldots$

(i) $\Rightarrow$ (ii). Since $f$ is weakly decreasing on $R$, then, for any vertex $v_{i}$ of $R$, there exist a unique edge $e_{i}$ of $R$ incident on it such that $f\left(v_{i}\right) \geq f\left(e_{i}\right)$. So condition (C1) of the definition of critical simplices does not hold. Reasoning similarly we have that, for any edge $e_{j}$ of $R$, there is one of its two vertices, $v_{j}$, such that $f\left(e_{j}\right) \leq f\left(v_{j}\right)$ and so, condition (C2) does not hold.

(ii) $\Rightarrow$ (iii). Let $v$ be a vertex of $R$. Since $v$ is not a critical vertex with respect to $f_{\mid R}$, by Proposition 1.5, $f(v)$ can not be a local minimum of $f_{\mid R}$. Moreover, by means of the comments before Proposition 1.5, if degree of $v$ is at least 2 then $f(v)$ can not be a local maximum of $f_{\mid R}$. Thus, only the first vertex of $R, v_{0}$, could be the unique local maximum of $f_{\mid R}$ (in the case $\left.f\left(v_{0}\right)>f\left(e_{0}\right)\right)$.

Now let $e$ be an edge of $R$. This edge is not critical with respect to $f_{\mid R}$ and hence, by Proposition 1.5, $f(e)$ can not be a local maximum of $f_{\mid R}$. Moreover, as we pointed out before Proposition 1.5, $f(e)$ can not be a local minimum of $f_{\mid R}$.

(iii) $\Rightarrow\left(\right.$ i). By using $($ iii $)$, we know that $f\left(e_{0}\right)$ is not a local maximum of $f_{\mid R}$ then it follows that either $f\left(v_{1}\right) \geq f\left(e_{0}\right)$ or $f\left(v_{0}\right) \geq f\left(e_{0}\right)$. If we assume that $f\left(v_{1}\right) \geq f\left(e_{0}\right)$, from (M2) we have $f\left(v_{1}\right) \geq f\left(e_{0}\right)>f\left(v_{0}\right)$, hence $f\left(v_{0}\right)$ is a local minimum of $f_{\mid R}$ but it is impossible by hypothesis. Now, let us consider the second possibility, that is, $f\left(v_{0}\right) \geq$ $f\left(e_{0}\right)$. By means of $(\mathrm{M} 2)$ we get $f\left(v_{0}\right) \geq f\left(e_{0}\right)>f\left(v_{1}\right)$ and since there is not any local minimum of $f_{\mid R}$ in $R$ then $f\left(v_{0}\right) \geq f\left(e_{0}\right)>f\left(v_{1}\right) \geq f\left(e_{1}\right)$. By repeating this procedure inductively we conclude that $f$ is weakly decreasing on $R$.

At this point, it is convenient to point out that $b_{1}(M)$, the first Betti number of a graph $M$, is the number of topologically independent cycles of $M$ or, equivalently, it is the number of chords (edges which are not in a given tree) with respect to a spanning tree.

Motivated by Example 2.1, we are going to study how the non existence of critical simplices and the topology of the graph are linked.

Theorem 2.5. Let $M$ be a connected graph. Then, $M$ admits a discrete Morse function without critical simplices if and only if $M$ is an infinite tree.

Proof. First, if there is a discrete Morse function defined on $M$ without critical simplices, it is clear that $M$ has to be infinite. Now, if $M$ contains at least one cycle, let $K$ be a finite subgraph of $M$ which contains at least one cycle of $M$. Then $\widehat{b}_{1} \geq 1$, where $\widehat{b}_{1}=\operatorname{dim}\left(H_{1}(K)\right)$. Using Theorem 1.2 , we get $\widehat{m}_{1}(f) \geq \widehat{b}_{1} \geq 1$, where $\widehat{m}_{1}(f)$ denotes the number of critical edges of the restriction of $f$ to $K$. By Proposition 1.3, we have that $m_{1}(f) \geq \widehat{m}_{1}(f) \geq \widehat{b}_{1}$ and this is a contradiction.

Conversely, suppose that $M$ is an infinite tree and let $v_{0}$ be a vertex of $M$. Then, let $R=v_{0}, e_{0}, v_{1}, e_{1}, \ldots$ be a ray in $M$ starting from $v_{0}$. First, we define the desired function $f$ in a weakly decreasing way on $R$, that is, such that:

$$
f\left(v_{0}\right) \geq f\left(e_{0}\right)>f\left(v_{1}\right) \geq f\left(e_{1}\right)>\ldots
$$


In the rest of $M$ we define $f$ weakly increasing when we are moving away from $v_{0}$ by paths (finite or not) different of ray $R$. To complete the definition on all $M$, we have to consider every path (finite or not again) starting from every vertex of $R$ which is not $v_{0}$. In this case we can repeat the same construction, that is, we define $f$ in a weakly increasing way when we are moving away from $R$.

Now, it is necessary to check that $f$ is a discrete Morse function with no critical simplices. Given a vertex $v$ in the ray $R$, there is only one edge $e$ (which is in $R$ ) incident with $v$ such that $f(v) \geq f(e)$ and the other edges $e^{\prime}$ incident with $v$ satisffy $f\left(e^{\prime}\right)>f(v)$.

If we consider now a vertex $w$ which is not in $R$, there is a unique path in $M-R$ connecting a vertex of $R$ and $w$. Then, by using the above construction, $f$ has to be weakly increasing on this path and so, there is an edge $\sigma$ incident with $w$ such that $f(\sigma) \leq f(w)$. Notice that $\sigma$ is the only edge verifying this condition because $f$ has been defined in a weakly increasing way as we are moving away from $R$.

Next, let $e$ an edge in the ray $R$. Since $f$ is weakly decreasing on $R$, its two vertices $v$ and $v^{\prime}$ verify either $f(v) \geq f(\sigma)>f\left(v^{\prime}\right)$ or $f(v)<f(\sigma) \leq f\left(v^{\prime}\right)$.

Finally, if $e$ is an edge which is not in $R$, since $f$ is weakly increasing as we are moving away from $R$, it holds either $f(v)<f(e) \leq f\left(v^{\prime}\right)$ or $f\left(v^{\prime}\right)<f(e) \leq f(v)$, where $v$ and $v^{\prime}$ denote the two vertices of $e$.

Consequently, $f$ is a discrete Morse function on $M$ without critical simplices.

Observe that Theorem 2.5 gives a method to define discrete Morse functions on infinite trees without critical simplices. Moreover, the constructions of such functions show that they are determined once a ray $R$ is fixed on the tree $M$. The following results establish a link between the non-existence of critical simplices and the existence of weakly decreasing rays.

Proposition 2.6. Let $M$ be a graph and $f$ be a discrete Morse function defined on $M$ with no critical vertices. Given a vertex $v$ of $M$, there is a unique weakly decreasing ray starting at $v$.

Proof. Since $f$ does not have critical vertices on $M$ then $M$ can not be a finite graph. Otherwise, by Theorem 1.2 (I2), it follows $m_{0}(f) \geq 1$. Let $v_{0}$ any vertex of $M$ which, obviously, is not a critical vertex. Then, there is a unique edge $e_{0}$ incident with $v_{0}$ such that $f\left(v_{0}\right) \geq f\left(e_{0}\right)$. Let $v_{1}$ the other vertex incident with $e_{0}$. As $f$ is a discrete Morse function, by $(\mathrm{M} 2), f\left(e_{0}\right)>f\left(v_{1}\right)$. Again, since $v_{1}$ is not a critical vertex, there is a unique edge $e_{1} \neq e_{0}$ such that $f\left(v_{1}\right) \geq f\left(e_{1}\right)$. Let $v_{2}$ be the other vertex of $e_{1}$. It is possible to repeat the same argument for $v_{2}$ and continue with this process indefinitely, getting a ray $R=v_{0}, e_{0}, v_{1}, e_{1}, v_{2}, e_{2}, \ldots$ on which $f$ is weakly decreasing. This construction does not stop after a finite number of steps. Otherwise, the last vertex $v_{n}$ verifies that there are not any edge $e$ such that $f\left(v_{n}\right) \geq f(e)$. Then, $f$ reaches a minimum on $v_{n}$ and so, $v_{n}$ is a critical vertex, which is a contradiction. Notice that in the above process we never repeat a vertex since the values of $f$ on the vertices of $R$ are strictly decreasing.

Now, if we suppose that there are two non-cofinal weakly decreasing rays, $R$ and $R^{\prime}$, starting at $v_{0}$, then, there is a vertex $v$ in both rays verifying that there is one edge $e$ in 
$R$ such that $f(v) \geq f(e)$ and there is one edge $e^{\prime}$ in $R^{\prime}$ such that $f(v) \geq f\left(e^{\prime}\right)$. This is a contradiction with (M1) and so, the ray $R$ is unique.

Corollary 2.7. Let $M$ be a connected graph and $f$ be a discrete Morse function defined on $M$ with no critical simplices. Then there is a unique (up to cofinality) decreasing ray in $M$.

Proof. By Theorem 2.5 we get that $M$ is an infinite tree. By using the above proposition, we know that for every vertex $v$, there is a weakly decreasing ray $R_{v}$ starting at $v$. Now, given any vertex $w \neq v$, let us see that $R_{v}$ and $R_{w}$ are cofinal. It is obvious if $w \in R_{v}$. So, let us suppose that $R_{v}$ and $R_{w}$ start at different vertices. Then we have two possibilities: either $R_{v}$ and $R_{w}$ have a common vertex and hence by (M1) they coincide from it (therefore they are cofinal) or they are disjoint. In this case, since there are no critical edges, there is a unique vertex $z$ joined with $v$ and $w$ by finite decreasing paths starting at $z$ and it is not possible by (M1).

\section{Generalized discrete Morse inequalities.}

In this section, we relate the topology of $M$, the numbers of critical vertices, critical edges and non-cofinal weakly decreasing rays of a discrete Morse function defined on $M$. This result generalizes the Forman's well known theorem for the finite case. It is interesting to point out that, despite the following result is restricted to discrete Morse functions with a finite number of critical simplices, this result holds for discrete Morse functions with an infinite number of critical simplices too. For this case, $(i)$ holds trivially and $(i i)$ is satisfied just by rewriting it as $b_{0}+m_{1}(f)=d_{0}+b_{1}+m_{1}(f)$.

Theorem 3.1. Let $M$ be an infinite graph and let $f$ be a discrete Morse function defined on $M$ such that $m_{0}(f)$ and $m_{1}(f)$ are finite. Then:

(i) $m_{0}(f)+d_{0} \geq b_{0}$ and $m_{1}(f) \geq b_{1}$, where $d_{0}$ is denoting the number of non-cofinal weakly decreasing rays on $M$.

(ii) $b_{0}-b_{1}=m_{0}(f)+d_{0}-m_{1}(f)$.

Proof. We observe that $m_{1}(f)<\infty$ implies that $M$ contains a finite number of cycles. Then, let $\bar{M}$ be a finite subgraph of $M$ such that it contains every critical simplex of $M$ and every cycle of $M$. Thus, $\bar{b}_{1}=b_{1}, \bar{m}_{0}(f) \geq m_{0}(f)$ and $\bar{m}_{1}(f)=m_{1}(f)$. When we restrict $f$ to the finite subgraph $\bar{M}$, we can apply the discrete Morse inequalities for the finite case (Theorem 1.2) and so, we get $m_{1}(f) \geq b_{1}$.

Now, if $b_{0}<+\infty$, it is possible to take $\bar{M}$ having the same number of connected components as $M$. Moreover, we can get $\bar{M}$ such that each of its connected components intersects a unique connected component of $M$. In fact, for each connected component of $M$ with critical simplices, it is enough to consider a connected and finite subgraph containing all critical simplices and all cycles which are included in the component. On the 
other hand, for each connected component of $M$ without critical simplices, since it has to be an infinite tree due to Theorem 2.5, it is enough to consider a finite subtree. Therefore, we get $\bar{b}_{0}=b_{0}$. Then, if $\bar{m}_{0}(f)=m_{0}(f)$, by using the discrete Morse inequalities for the finite case on $\bar{M}$, we obtain:

$$
m_{0}(f)+d_{0} \geq m_{0}(f)=\bar{m}_{0}(f) \geq \bar{b}_{0}=b_{0} .
$$

Next, if $\bar{m}_{0}(f)>m_{0}(f)$, there exist critical vertices with respect to the restriction of $f$ to $\bar{M}$ which are not critical with respect to $f$. Then, every vertex of this kind is in a weakly decreasing ray in $M$. In fact, let $v_{0}$ be a critical vertex with respect to the restriction of $f$ to $\bar{M}$ which is not critical with respect to $f$. This implies that there exists a unique edge $e_{0}$ incident with $v_{0}$ and belonging to $M-\bar{M}$ such that $f\left(v_{0}\right) \geq f\left(e_{0}\right)$. Since $e_{0}$ does not belong to $\bar{M}$, it can not be critical with respect to $M$ and thus, its other vertex $v_{1}$ satisfies $f\left(v_{1}\right)<f\left(e_{0}\right)$. Moreover, $v_{1}$ is not in $\bar{M}$ because, in other case, $e_{0}$ is in $\bar{M}$ and it is impossible. So, $v_{1}$ is not a critical vertex with respect to $f$ and, consequently, there is a unique edge $e_{1}$ in $M-\bar{M}$ (that is, not critical with respect to $f$ ) and incident with $v_{1}$, such that $f\left(e_{1}\right)>f\left(v_{2}\right)$, where $v_{2}$ is the other vertex of $e_{1}$. If we suppose that $v_{2}$ is in $\bar{M}$, then we find two different paths from $v_{0}$ to $v_{2}$ : one through $\bar{M}$, since $v_{0}$ and $v_{2}$ are in the same connected component of $\bar{M}$ and the other determined by our construction: $v_{0}, e_{0}, v_{1}, e_{1}, v_{2}$, which is out of $\bar{M}$. If we join both paths we get a cycle which is not in $\bar{M}$ and this is impossible. So, $v_{2}$ is in $M-\bar{M}$.

The path $v_{0}, e_{0}, v_{1}, e_{1}, v_{2}, \ldots$ can be extended indefinitely, so it is a ray. Otherwise, its last vertex $v_{n}$ would have to verify one of these two conditions: either $v_{n}$ is a critical vertex with respect to $f$ or $v_{n}$ is in $\bar{M}$. The first option is impossible by definition of $\bar{M}$ and the second is impossible too, because reasoning in a similar way as before, it would imply that we could get a cycle which is not in $\bar{M}$.

At this point, we know that every critical vertex with respect to the restriction of $f$ to $\bar{M}$ which is not critical with respect to $f$ is in a weakly decreasing ray. Conversely, given a weakly decreasing ray $R$ in $M-\bar{M}$ there is a unique vertex in $\bar{M}$ joined to $R$ by a finite decreasing path. This can proved as follows: since every connected component of $\bar{M}$ intersects a unique component of $M$ and there are no critical edges with respect to $f$ in $M-\bar{M}$, then there is a finite decreasing path $\gamma_{v}$ starting from a vertex $v \in \bar{M}$, which is critical with respect to the restriction of $f$ to $\bar{M}$ but not with respect to $f$, and ending at a vertex of $R$. Notice that $v$ is unique since otherwise there is a cycle in $M$ not contained in $\bar{M}$. We conclude by joining $R$ and $\gamma_{v}$ to obtain a new weakly decreasing ray $R^{\prime}$ starting at a vertex in $\bar{M}$ which is cofinal with $R$.

Now, If we apply discrete Morse inequalities for the finite case on $\bar{M}$, then we can replace every critical vertex with respect to the restriction of $f$ to $\bar{M}$ but not with respect to $f$ by its corresponding weakly decreasing ray. Thus, the total number of critical vertices with respect to the restriction of $f$ to $\bar{M}$ can be expressed as the sum of the number of critical vertices with respect to $f$ and the number of non-cofinal weakly decreasing rays on $M$. That is, $\bar{m}_{0}(f)=m_{0}(f)+d_{0}$ and we get the desired equalities and inequalities.

Finally, if $b_{0}=+\infty$, since $\bar{M}$ is a finite subgraph of $M$, it only contains a finite number of components of $M$ and then, there is an infinite number of connected components of $M$ 
not in $\bar{M}$. All of these components do not have critical simplices and then, all of them are infinite trees, every single one of them having a unique weakly decreasing ray. So, the number of non cofinal weakly decreasing rays is infinite and then not only the inequalities, but also the equality holds trivially.

\section{Characterizing graphs by the number of critical el- ements.}

As an application of generalized discrete Morse inequalities proved in Section 3, we are going to characterize an infinite graph $M$ attending to the numbers of critical simplices and weakly decreasing rays of discrete Morse functions defined on $M$.

In order to unify the concepts of critical simplex ans weakly decreasing ray, we shall start by introducing the notion of critical element.

Definition 4.1. Let $M$ be an infinite graph and let $f$ be a discrete Morse function defined on $G$. Acritical element is either a critical simplex of $f$ on $M$ or a weakly decreasing ray.

Then we have:

Theorem 4.2. An infinite connected graph $M$ admits a discrete Morse function with $n$ critical elements if and only if:

(i) If $n$ is odd, then $M$ is either a tree or $M=G \cup F$, where $G$ is a finite subgraph which is the union of an even number of (topological) independent cycles and $F$ is a forest.

(ii) If $n$ is even, $M=G \cup F$ where $G$ is a finite subgraph which is the union of an odd number of (topological) independent cycles and $F$ is a forest.

Proof. Let us suppose that $M$ admits a discrete Morse function with $n$ critical elements. Observe that, from Theorem 3.1, $m_{0}+d_{0} \geq b_{0}(M)=1$ and $b_{1}(M)=1-2\left(m_{0}+d_{0}\right)+n$.

First, let $n=2 k+1$. If $b_{1}(M)=0$, then $M$ is a tree. If $b_{1}(M)>0$, then $b_{1}(M)=2\left(k+1-m_{0}-d_{0}\right)$. Consequently, $M=G \cup F$, where $G$ is the union of the $2\left(k+1-m_{0}-d_{0}\right)$ span cycles and $F=M-G$.

On the other hand, let $n=2 k$. Then, $b_{1}(M)=1+2\left(k-m_{0}-d_{0}\right) \geq 1$. So, $M=G \cup F$, where $G$ is the union of the $2\left(k-m_{0}-d_{0}\right)+1$ span cycles and $F=M-G$.

Conversely, let $M$ be a graph such that $M=G \cup F$, where $G$ is a finite subgraph which is the union of $m$ independent cycles and $F$ is a forest (obviously with, at least, one infinite connected component). 
Then, we can define a discrete Morse function $f$ on $M$ with $n$ critical elements, being $n$ odd if $m$ is even and even if $m$ is odd. First, since $M$ is connected, there has to be, at least, either a critical vertex or a weakly decreasing ray on $M$. Thus, we define $f$ by fixing such a critical element. Now, we select a critical edge (that is, we define $f$ in such a way that this edge is critical one) in every cycle of $G$. At this step, $M$ has $b_{1}(M)+1$ critical elements. To get more critical elements, we select new critical edges. Notice that, since we are defining $f$ as a discrete Morse function, from Theorem 3.1, the appearance of each new critical edge must imply the appearance of either a new critical vertex or another weakly decreasing ray non-cofinal with the above considered ones.

In the rest of $M$, we define $f$ in such a way that no new critical elements appear. So, $n=1+b_{1}(M)+2 k$ and this completes the proof.

Notice that Theorem 2.5 can be considered as a particular case of the above theorem for $n=2 k+1$ with $k=0$.

It is interesting to point out that we can obtain a version of the above theorem for the finite case, that is, a characterization of finite connected graphs by means of the number of critical simplices of discrete Morse functions defined on it. This can be carried out essentially by repeating the above proof without considering weakly decreasing rays.

ACKNOWLEDGEMENT. We thank to the referee for his helpful comments and suggestions which greatly improved this paper.

\section{References}

[1] R. Forman. Morse Theory for Cell Complexes. Advances in Mathematics. vol.134 pp.90-145. 1998.

[2] T. Lewiner, H. Lopes and G. Tavares. Towards optimality in discrete Morse theory. Experimental Mathematics. vol. 12 (3) pp.271-285. 2003

[3] R. Ayala, L.M. Fernández and J.A. Vilches. Morse inequalities on certain infinite 2-complexes. Glasgow Math. J. vol 49 (2007) 155-165.

[4] R. Diestel. Graph Theory. Graduate texts in mathematics, 173, Springer-Verlag, New York, 1997. Zbl 0945.05002

[5] J. Milnor. Morse Theory. Annals of Mathematics Study 51, Princeton Univ. Press, Princeton, NJ, 1962. 